\title{
A Precise Asymptotic Behaviour of the Large Deviation Probabilities for Weighted Sums
}

\author{
Gooty Divanji, Kokkada Vidyalaxmi \\ Department of Statistics, Manasagangothri University of Mysore, Mysore, India \\ E-mail: gootydivan@yahoo.co.in,vidyalaxmi.k@gmail.com \\ Received June 11, 2011; revised July 1, 2011; accepted July 8, 2011
}

\begin{abstract}
Let $\left\{\mathrm{X}_{\mathrm{n}}, \mathrm{n} \geq 1\right\}$ be a sequence of independent and identically distributed positive valued random variables with a common distribution function $\mathrm{F}$. When $\mathrm{F}$ belongs to the domain of partial attraction of a semi stable law with index $\alpha, 0<\alpha<1$, an asymptotic behavior of the large deviation probabilities with respect to properly normalized weighted sums have been studied and in support of this we obtained Chover's form of law of iterated logarithm.
\end{abstract}

Keywords: Large Deviations, Law of Iterated Logarithm, Semi-Stable Law, Domain of Partial Attraction, Weighted Sums

\section{Introduction}

Let $\left\{X_{n}, n \geq 1\right\}$ be a sequence of independent and identically distributed (i.i.d) positive valued random variables (r.v.s) with a common distribution function F. Let BV $[0,1]$ be the set of all continuous bounded variation functions over $[0,1]$. Set

$\mathrm{S}_{\mathrm{n}}=\sum_{\mathrm{k}=1}^{\mathrm{n}} \mathrm{X}_{\mathrm{k}}, \mathrm{n} \geq 1$, and $\mathrm{T}_{\mathrm{n}}=\sum_{\mathrm{k}=1}^{\mathrm{n}} \mathrm{f}\left(\frac{\mathrm{k}}{\mathrm{n}}\right) \mathrm{X}_{\mathrm{k}}$,

where $\mathrm{f}$ is a member of $\mathrm{BV}[0,1]$. Let $\left\{\mathrm{n}_{\mathrm{k}}, \mathrm{k} \geq 1\right\}$ be a strictly increasing subsequence of positive integers such that $\frac{\mathrm{n}_{\mathrm{k}+1}}{\mathrm{n}_{\mathrm{k}}} \rightarrow \mathrm{r}(\geq 1)$ as $\mathrm{k} \rightarrow \infty$. Kruglov [1] established that, if there exists sequences $\left(a_{k}\right)$ and $\left(b_{k}\right)$ of real constants, $b_{k} \rightarrow \infty$ as $\mathrm{k} \rightarrow \infty$, such that

$$
\operatorname{Lim}_{\mathrm{k} \rightarrow \infty} \mathrm{P}\left(\frac{\mathrm{S}_{\mathrm{n}_{\mathrm{k}}}}{\mathrm{b}_{\mathrm{k}}}-\mathrm{a}_{\mathrm{k}} \leq \mathrm{x}\right)=\mathrm{G}_{\alpha}(\mathrm{x})
$$

at all continuity points $\mathrm{x}$ of $\mathrm{G}_{\alpha}$, then $\mathrm{G}_{\alpha}$ is necessarily a semi stable d.f with characteristic exponent $\alpha, 0<\alpha \leq 2$. When $\alpha=2$, semi-stable becomes normal.

It is known that probabilities of the type $P\left(\left|S_{n}\right|>x_{n}\right)$, or either of the one sided components, are called large deviation probabilities, where $\left\{\mathrm{x}_{\mathrm{n}}, \mathrm{n} \geq 1\right\}$ is a monotone sequence of positive numbers with $\mathrm{x}_{\mathrm{n}} \rightarrow \infty$ as $\mathrm{n} \rightarrow \infty$ such that $\frac{S_{n}}{x_{n}} \stackrel{p}{\longrightarrow} 0$. In fact, under different conditions on sequence of r.v.s, Heyde [2-4] studied the large deviation problems for partial sums. In brief, for the r.v.s which are in the domain of attraction of a stable law and r.v.s which are not belong to the domain of partial attraction of the normal law, Heyde [2] and [3] established the order of magnitude of the larger deviation probabilities, where as in Heyde [3], he obtained the precise asymptotic behavior of large deviation probabilities for r.v.s in the domain of attraction of stable law.

When r.v.s. has i.i.d symmetric stable r.v.s, Chover [5] obtained the law of iterated logarithm (LIL) for partial sums by normalizing in the power and for r.v.s which are in the domain of attraction of a stable law, Peng and Qi [6] obtained Chover's type LIL for weighted sums, where the weights are belongs to BV[0, 1]. Many authors studied the non-trivial limit behavior for different weighted sums. See Peng and Qi [6] and references therein.

Probability of large values plays an important role in studying non-trivial limit behavior for stable like r.v.s. As far as properly normalized partial sums of stable like r.v.s, we can use the asymptotic results of Heyde [2-4]. (See Divanji [7]). However the observations made by Heyde [2-4] on the large deviation probabilities implicitly motivated us to study the large deviation probabilities for weighted sums. In fact, when the underlying i.i.d positive valued r.v.s are in the domain of partial attraction of a semi stable law of Kruglov's [1] setup, denoted as $\mathrm{F} \in \mathrm{DP}(\alpha), 0<\alpha<1$, a precise asymptotic behavior 
of the large deviation probabilities of Heyde [2-4] can be obtained for weighted sums. In support of this can be considered for Chover's type of non-trivial limit behavior for weighted sums.

In the next section we present some lemmas and main result in Section 3. In the last section, we discuss the existence of Chover's form of LIL for weighted sums. In the process i.o, a.s and s.v. mean 'infinitely often', 'almost surely' and 'slowly varying' respectively. C, $\varepsilon, \mathrm{k}$ and $\mathrm{n}$ with or without a super script or subscript denote positive constants with $\mathrm{k}$ and $\mathrm{n}$ confined to be integers. In the sequel, observe that when $\alpha<1, \mathrm{a}_{\mathrm{k}}$ can always be chosen to be zero.

\section{Lemmas}

\section{Lemma 2.1}

Let $\mathrm{F} \in \mathrm{DP}(\alpha), 0<\alpha<1$. Then there exists s. v. function $\mathrm{L}$, such that

$$
\operatorname{Lim}_{x \rightarrow \infty} \frac{x^{\alpha}(1-F(x))}{L(x)}=1 .
$$

\section{Lemma 2.2}

Let $\mathrm{F} \in \mathrm{DP}(\alpha), 0<\alpha<1$ and let

$B_{n}=\inf \left\{x>0: 1-F(x) \geq \frac{1}{n}\right\}$. Then $B_{n}=n^{1 / \alpha} 1(n)$,

where 1 is a function s. v. at $\infty$.

The above lemmas can be referred to Divanji and Vasudeva [8].

\section{Lemma 2.3}

Let $\mathrm{L}$ be any s. v. function and let $\left(\mathrm{x}_{\mathrm{n}}\right)$ and $\left(\mathrm{y}_{\mathrm{n}}\right)$ be sequence of real constants tending to $\infty$ as $n \rightarrow \infty$. Then for any $\delta>0, \operatorname{Lim}_{\mathrm{n} \rightarrow \infty} \mathrm{y}_{\mathrm{n}}^{\delta} \frac{\mathrm{L}\left(\mathrm{x}_{\mathrm{n}} \mathrm{y}_{\mathrm{n}}\right)}{\mathrm{L}\left(\mathrm{x}_{\mathrm{n}}\right)}=\infty$ and $\operatorname{Lim}_{n \rightarrow \infty} y_{n}^{-\delta} \frac{L\left(x_{n} y_{n}\right)}{L\left(x_{n}\right)}=0$.

This lemma can be referred to Drasin and Seneta [9].

\section{Lemma 2.4}

Let $\mathrm{F} \in \mathrm{DP}(\alpha), 0<\alpha<1$. Let $\left(\mathrm{x}_{\mathrm{n}}\right)$ be a monotone sequence of real numbers tending to $\infty$ as $n \rightarrow \infty$ and $B_{n}$ defined in Lemma 2.2. Then $\mathrm{B}_{\mathrm{n}}^{-1} \mathrm{x}_{\mathrm{n}}^{-1} \mathrm{~S}_{\mathrm{n}} \stackrel{\mathrm{p}}{\longrightarrow} 0$, as $\mathrm{n} \rightarrow \infty$.

This lemma can be referred to Vasudeva and Divanji [10].

\section{Lemma 2.5}

Let $\mathrm{F} \in \mathrm{DP}(\alpha), 0<\alpha<1$. Let $\left(\mathrm{x}_{\mathrm{n}}\right)$ be a monotone sequence of real numbers tending to $\infty$ as $n \rightarrow \infty$ and $B_{n}$ defined in lemma 2. Then $\mathrm{x}_{\mathrm{n}}^{-1} \mathrm{~B}_{\mathrm{n}}^{-1} \mathrm{~T}_{\mathrm{n}} \stackrel{\mathrm{p}}{\longrightarrow} 0$ as $\mathrm{n} \rightarrow \infty$, with $B_{n}$ defined in Lemma 2.2.

\section{Proof}

Since $f \in B V[0,1]$. Hence there exists a constant $C$ such that $|f(x)| \leq C$ and $\sum_{k=1}^{n-1}\left|f\left(\frac{k}{n}\right)-f\left(\frac{k+1}{n}\right)\right| \leq C$, for all $\mathrm{n} \geq 1$.

Therefore

$$
\begin{aligned}
T_{n} & =\sum_{k=1}^{n} f\left(\frac{k}{n}\right) X_{k} \\
& =\sum_{k=1}^{n-1}\left(f\left(\frac{k}{n}\right)-f\left(\frac{k+1}{n}\right)\right) S_{k}+f(1) S_{n} \leq 2 C \max _{1 \leq k \leq n} S_{k}
\end{aligned}
$$

Dividing on both sides by $\mathrm{x}_{\mathrm{n}} \mathrm{B}_{\mathrm{n}}$, we have

$\frac{T_{n}}{x_{n} B_{n}} \leq 2 C \max _{1 \leq k \leq n} \frac{S_{k}}{X_{n} B_{n}}$ Observe that $X_{i}$ 's are i.i.d positive valued r.v.s which are in the domain of partial attraction of a semi-stable law and hence

$\max _{1 \leq \mathrm{k} \leq \mathrm{n}} \frac{\mathrm{S}_{\mathrm{k}}}{\mathrm{X}_{\mathrm{n}} \mathrm{B}_{\mathrm{n}}} \leq \frac{\mathrm{S}_{\mathrm{n}}}{\mathrm{x}_{\mathrm{n}} \mathrm{B}_{\mathrm{n}}}$ and by lemma 2.4 we have $\frac{S_{n}}{x_{n} B_{n}} \stackrel{p}{\longrightarrow} \infty$. This gives $\frac{T_{n}}{x_{n} B_{n}} \stackrel{p}{\longrightarrow} 0$, as $n \rightarrow \infty$.

\section{Main Results}

\section{Theorem 3.1}

Let $\mathrm{F} \in \mathrm{DP}(\alpha), 0<\alpha<1$. Let $\left(\mathrm{x}_{\mathrm{n}}\right)$ be a monotone sequence of real numbers tending to $\infty$ as $n \rightarrow \infty$ and $B_{n}$ defined in lemma 2.2. Then $\operatorname{Lim}_{n \rightarrow \infty} \frac{P\left(T_{n} \geq x_{n} B_{n}\right)}{n P\left(X \geq x_{n} B_{n}\right)}=1$.

\section{Proof}

To prove the assertion, it is enough to show that

$$
\begin{aligned}
& 0<\underset{n \rightarrow \infty}{\operatorname{Liminf}} \frac{P\left(T_{n} \geq x_{n} B_{n}\right)}{n P\left(X \geq x_{n} B_{n}\right)} \\
& \leq \operatorname{LimSup}_{n \rightarrow \infty} \frac{P\left(T_{n} \geq x_{n} B_{n}\right)}{n P\left(X \geq x_{n} B_{n}\right)}<\infty .
\end{aligned}
$$

Let $\varepsilon>0$ and define $A_{i}=\left\{f\left(\frac{i}{n}\right) X_{i} \geq(1+\varepsilon) x_{n} B_{n}\right\}$ and $\mathrm{B}_{\mathrm{i}}=\left\{\sum_{\mathrm{j}=1, \mathrm{j} \neq \mathrm{i}}^{\mathrm{n}} \mathrm{f}\left(\frac{\mathrm{j}}{\mathrm{n}}\right) \mathrm{X}_{\mathrm{j}} \leq \varepsilon \mathrm{x}_{\mathrm{n}} \mathrm{B}_{\mathrm{n}}\right\}, i=1,2, \cdots, n$.

Proceeding on the lines of Heyde [4] and Lemma 3.1 of Vasudeva [12], we get,

$$
\begin{aligned}
& \mathrm{P}\left(\mathrm{T}_{\mathrm{n}} \geq \mathrm{x}_{\mathrm{n}} \mathrm{B}_{\mathrm{n}}\right) \geq \sum_{\mathrm{i}=1}^{\mathrm{n}} \mathrm{P}\left(\mathrm{A}_{\mathrm{i}}\right)\left(\mathrm{P}\left(\mathrm{B}_{\mathrm{i}}\right)-\sum_{\mathrm{j}=1}^{\mathrm{n}} \mathrm{P}\left(\mathrm{A}_{\mathrm{j}}\right)\right) \\
& \geq \mathrm{nP}\left(\mathrm{A}_{1}\right)\left[\mathrm{P}\left(\mathrm{B}_{1}\right)-\mathrm{nP}\left(\mathrm{A}_{1}\right)\right]
\end{aligned}
$$

From Lemma 2.5, we have $\frac{\mathrm{T}_{\mathrm{n}}}{\mathrm{x}_{\mathrm{n}} \mathrm{B}_{\mathrm{n}}} \stackrel{\mathrm{p}}{\longrightarrow} 0$, as $\mathrm{n} \rightarrow \infty$ and given $\delta>0$ with $1-2 \delta>0$, we can choose $\mathrm{N}_{1}$ so 
large such that $\mathrm{P}\left(\mathrm{B}_{\mathrm{i}}\right)>1-2 \delta$ for all $\mathrm{n} \geq \mathrm{N}_{1}$ and for all $i=1,2,3, \cdots, n$. Further from Lemma 2.5 , we see that $\mathrm{nP}\left(\mathrm{A}_{\mathrm{i}}\right) \rightarrow 0$ as $\mathrm{n} \rightarrow \infty$, so that we can choose $\mathrm{N}_{2}$ so large that $\mathrm{n} \mathrm{P}\left(\mathrm{A}_{\mathrm{i}}\right)<\delta$, for $\mathrm{n} \geq \mathrm{N}_{2}$. Thus for $\mathrm{n} \geq \mathrm{N}=\max \left(\mathrm{N}_{1}\right.$, $\mathrm{N}_{2}$ ), we obtain from (1),

$\mathrm{P}\left(\mathrm{T}_{\mathrm{n}} \geq \mathrm{x}_{\mathrm{n}} \mathrm{B}_{\mathrm{n}}\right) \geq \mathrm{n}(1-2 \delta) \mathrm{P}\left(\mathrm{X} \geq(1+\varepsilon) \mathrm{x}_{\mathrm{n}} \mathrm{B}_{\mathrm{n}}\right)$, this implies

$$
\begin{aligned}
& \frac{\mathrm{P}\left(\mathrm{T}_{\mathrm{n}} \geq \mathrm{x}_{\mathrm{n}} \mathrm{B}_{\mathrm{n}}\right)}{\mathrm{nP}\left(\mathrm{X} \geq \mathrm{x}_{\mathrm{n}} \mathrm{B}_{\mathrm{n}}\right)} \geq \frac{\mathrm{n}(1-2 \delta) \mathrm{P}\left(\mathrm{X} \geq(1+\varepsilon) \mathrm{x}_{\mathrm{n}} \mathrm{B}_{\mathrm{n}}\right)}{\mathrm{nP}\left(\mathrm{X} \geq \mathrm{x}_{\mathrm{n}} \mathrm{B}_{\mathrm{n}}\right)} . \\
& \geq(1-2 \delta) \frac{\mathrm{P}\left(\mathrm{X} \geq(1+\varepsilon) \mathrm{x}_{\mathrm{n}} \mathrm{B}_{\mathrm{n}}\right)}{\mathrm{P}\left(\mathrm{X} \geq \mathrm{x}_{\mathrm{n}} \mathrm{B}_{\mathrm{n}}\right)}
\end{aligned}
$$

Using Lemma 2.1, we have

$$
\begin{aligned}
& \frac{\mathrm{P}\left(\mathrm{T}_{\mathrm{n}} \geq \mathrm{x}_{\mathrm{n}} \mathrm{B}_{\mathrm{n}}\right)}{\mathrm{nP}\left(\mathrm{X} \geq \mathrm{x}_{\mathrm{n}} \mathrm{B}_{\mathrm{n}}\right)} \geq \frac{(1-2 \delta)}{(1+\varepsilon)^{\alpha}} \frac{\mathrm{L}\left((1+\varepsilon) \mathrm{x}_{\mathrm{n}} \mathrm{B}_{\mathrm{n}}\right)}{\mathrm{x}_{\mathrm{n}}^{\alpha} \mathrm{B}_{\mathrm{n}}^{\alpha}} \frac{\mathrm{x}_{\mathrm{n}}^{\alpha} \mathrm{B}_{\mathrm{n}}^{\alpha}}{\mathrm{L}\left(\mathrm{x}_{\mathrm{n}} \mathrm{B}_{\mathrm{n}}\right)} \\
& \geq \frac{(1-2 \delta)}{(1+\varepsilon)^{\alpha}} \frac{\mathrm{L}\left((1+\varepsilon) \mathrm{x}_{\mathrm{n}} \mathrm{B}_{\mathrm{n}}\right)}{\mathrm{L}\left(\mathrm{x}_{\mathrm{n}} \mathrm{B}_{\mathrm{n}}\right)}
\end{aligned}
$$

Choose $\varepsilon>0$ sufficiently very small such that $\operatorname{Lim}_{n \rightarrow \infty} \frac{L\left((1+\varepsilon) x_{n} B_{n}\right)}{L\left(x_{n} B_{n}\right)}=1$, one can find a constant $C_{1}>0$ such that

$$
\operatorname{Liminf}_{n \rightarrow \infty} \frac{P\left(T_{n} \geq x_{n} B_{n}\right)}{n P\left(X \geq x_{n} B_{n}\right)}>C_{1}>0 .
$$

In order to complete the proof, we use truncation method.

Define

$$
Y_{k}= \begin{cases}X_{k}, & \text { if } f\left(\frac{k}{n}\right) X_{k} \leq X_{n} B_{n} \\ 0, & \text { otherwise }\end{cases}
$$

Let $R_{k}=f\left(\frac{k}{n}\right) X_{k}-f\left(\frac{k}{n}\right) Y_{k}$,

$T_{1, n}=\sum_{k=1}^{n} f\left(\frac{k}{n}\right) Y_{k}$ and $T_{2, n}=\sum_{k=1}^{n} R_{k}$. Notice that

$\mathrm{P}\left(\mathrm{T}_{\mathrm{n}} \geq \mathrm{x}_{\mathrm{n}} \mathrm{B}_{\mathrm{n}}\right) \leq \mathrm{P}\left(\mathrm{T}_{1, \mathrm{n}}>\mathrm{x}_{\mathrm{n}} \mathrm{B}_{\mathrm{n}}\right)+\mathrm{P}\left(\mathrm{T}_{2, \mathrm{n}} \neq 0\right)$. This implies

$$
\frac{P\left(T_{n} \geq x_{n} B_{n}\right)}{n P\left(X \geq x_{n} B_{n}\right)} \leq \frac{P\left(T_{1, n} \geq x_{n} B_{n}\right)}{n P\left(X \geq x_{n} B_{n}\right)}+\frac{P\left(T_{2, n} \neq 0\right)}{n P\left(X \geq x_{n} B_{n}\right)}
$$

Observe that

$\mathrm{P}\left(\mathrm{T}_{2, \mathrm{n}} \neq 0\right) \leq \mathrm{nP}\left(\mathrm{R}_{1} \neq 0\right)=\mathrm{nP}\left(\mathrm{f}\left(\frac{1}{\mathrm{n}}\right) \mathrm{X} \geq \mathrm{x}_{\mathrm{n}} \mathrm{B}_{\mathrm{n}}\right)$, for

fixed $\mathrm{n}$ and $\mathrm{f}$ is continuous $\mathrm{BV}[0,1]$ and it attains bounds. Hence using Lemma 1, we have,

$$
\begin{aligned}
& \frac{P\left(T_{2, n} \neq 0\right)}{n P\left(X \geq x_{n} B_{n}\right)}=\frac{n P\left(f\left(\frac{1}{n}\right) X \geq x_{n} B_{n}\right)}{n P\left(X \geq x_{n} B_{n}\right)} \\
& \leq \frac{L\left(\frac{x_{n} B_{n}}{f\left(\frac{1}{n}\right)}\right)}{x_{n}^{\alpha} B_{n}^{\alpha}} \frac{x_{n}^{\alpha} B_{n}^{\alpha}}{L\left(x_{n} B_{n}\right)}\left(f\left(\frac{1}{n}\right)\right)^{\alpha} \\
& \leq\left(f\left(\frac{1}{n}\right)\right)^{\alpha} \frac{L\left(\frac{x_{n} B_{n}}{f\left(\frac{1}{n}\right)}\right)}{L\left(x_{n} B_{n}\right)} .
\end{aligned}
$$

Using Karamata's representation of s.v. function, one gets that

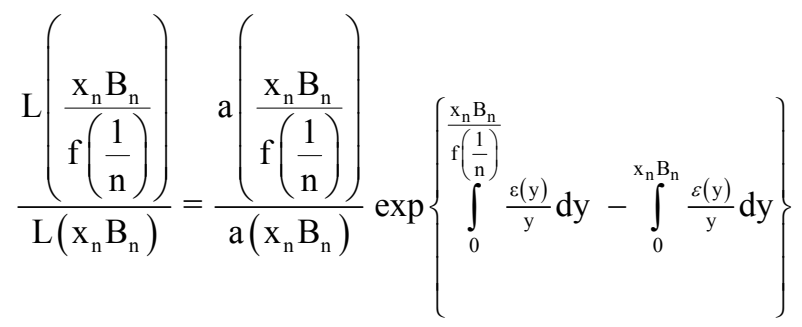

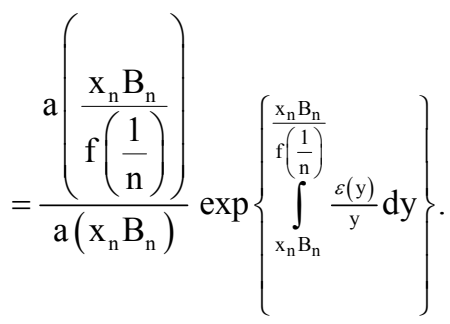

Since $\mathrm{a}(\mathrm{x}) \rightarrow \mathrm{C}$ as $\mathrm{x} \rightarrow \mathrm{C}$ and $\delta(\mathrm{y}) \rightarrow 0$ as $\mathrm{y} \rightarrow \infty$, there exists $\mathrm{C}_{0}>0$ and $\delta_{0}<\alpha$, such that

$$
\frac{a\left(\frac{x_{n} B_{n}}{f\left(\frac{1}{n}\right)}\right)}{a\left(x_{n} B_{n}\right)} \leq C_{0}, \varepsilon(y) \leq \delta_{0} \text {, for } y \geq x_{n} B_{n} \text {. }
$$

This yield

$$
\frac{\mathrm{L}\left(\frac{\mathrm{x}_{\mathrm{n}} \mathrm{B}_{\mathrm{n}}}{\mathrm{f}\left(\frac{1}{\mathrm{n}}\right)}\right)}{\mathrm{L}\left(\mathrm{x}_{\mathrm{n}} \mathrm{B}_{\mathrm{n}}\right)} \leq \mathrm{C}_{0} \exp \left\{\delta_{0} \log \left(\frac{1}{\left.\mathrm{f}\left(\frac{1}{\mathrm{n}}\right)\right)}\right)\right\} \leq \frac{\mathrm{C}_{0}}{\left(\mathrm{f}\left(\frac{1}{\mathrm{n}}\right)\right)^{\delta_{0}}} .
$$

Substituting (4) in (3), one can find some constant $C_{1}$ such that the second term in (2) becomes 
$\frac{\mathrm{P}\left(\mathrm{T}_{2, \mathrm{n}} \neq 0\right)}{\mathrm{nP}\left(\mathrm{X} \geq \mathrm{x}_{\mathrm{n}} \mathrm{B}_{\mathrm{n}}\right)} \leq \mathrm{C}_{1}\left(\mathrm{f}\left(\frac{1}{\mathrm{n}}\right)\right)^{\alpha-\delta}$. Since $\mathrm{f} \in \mathrm{BV}[0,1]$ and $\mathrm{f}\left(\frac{1}{\mathrm{n}}\right) \rightarrow \mathrm{f}(0)(\in \mathrm{BV}[0,1])$, as $\mathrm{n} \rightarrow \infty$. Therefore we can find some constant $\mathrm{C}_{2}\left(>\mathrm{C}_{1}\right)$ such that

$$
\operatorname{Lim}_{n \rightarrow \infty} \frac{P\left(T_{2, n} \neq 0\right)}{n P\left(X \geq x_{n} B_{n}\right)} \leq C_{2}<\infty .
$$

Now consider the first term in the right of (2). By Tchebychev's inequality, we get

$$
\frac{P\left(T_{1, n} \geq x_{n} B_{n}\right)}{n P\left(X \geq x_{n} B_{n}\right)} \leq \frac{E\left(T_{1, n}^{2}\right)}{n x_{n}^{2} B_{n}^{2} P\left(X \geq x_{n} B_{n}\right)} .
$$

Since

$$
E\left(T_{1, n}^{2}\right)=\sum_{k=1}^{n} f^{2}\left(\frac{k}{n}\right) E Y_{k}^{2}+\sum_{k=1}^{n} \sum_{m=1}^{n} f\left(\frac{k}{n}\right) f\left(\frac{m}{n}\right) E Y_{k} E Y_{m},
$$

for $\mathrm{k} \neq \mathrm{m}$. Hence

$$
\begin{aligned}
& \frac{P\left(T_{1, n} \geq x_{n} B_{n}\right)}{n P\left(X \geq x_{n} B_{n}\right)} \leq \frac{E\left(T_{1, n}^{2}\right)}{n x_{n}^{\alpha} B_{n}^{\alpha} P\left(X \geq x_{n} B_{n}\right)} \\
& =\frac{\sum_{k=1}^{n} f^{2}\left(\frac{k}{n}\right) E Y_{k}^{2}}{n x_{n}^{\alpha} B_{n}^{\alpha} P\left(X \geq x_{n} B_{n}\right)}+\frac{\sum_{k=1}^{n} \sum_{m=1}^{n} f\left(\frac{k}{n}\right) f\left(\frac{m}{n}\right) E Y_{k} E Y_{m}}{n x_{n}^{\alpha} B_{n}^{\alpha} P\left(X \geq x_{n} B_{n}\right)} .
\end{aligned}
$$

By Theorem 1, on page 544, of Feller [12] and Lemma 2.1 , one gets that

$$
\begin{aligned}
& \frac{\sum_{k=1}^{n} f^{2}\left(\frac{k}{n}\right) E Y_{k}^{2}}{n x_{n}^{2} B_{n}^{2} P\left(X \geq x_{n} B_{n}\right)} \\
& \leq \frac{x_{n}^{\alpha} B_{n}^{\alpha} \sum_{k=1}^{n} f^{2}\left(\frac{k}{n}\right) \frac{x_{n}^{2-\alpha} B_{n}^{2-\alpha}}{f^{2-\alpha}\left(\frac{k}{n}\right)} L\left(\frac{x_{n} B_{n}}{f\left(\frac{k}{n}\right)}\right)}{n x_{n}^{2} B_{n}^{2} L\left(x_{n} B_{n}\right)} \\
& \leq \frac{x_{n}^{\alpha} B_{n}^{\alpha} \sum_{k=1}^{n} f^{\alpha}\left(\frac{k}{n}\right) x_{n}^{2-\alpha} B_{n}^{2-\alpha} L\left(\frac{x_{n} B_{n}}{n\left(\frac{k}{n}\right)}\right)}{n x_{n}^{2} B_{n}^{2} L\left(x_{n} B_{n}\right)} \\
& \leq \frac{1}{n} \sum_{k=1}^{n} f^{\alpha}\left(\frac{k}{n}\right) \frac{L\left(\frac{x_{n} B_{n}}{L\left(\frac{k}{n}\right)}\right)}{L\left(x_{n} B_{n}\right)} .
\end{aligned}
$$

Using similar steps of (4), one can find some constant $\mathrm{C}_{3}$ such that $\frac{\sum_{k=1}^{n} f^{2}\left(\frac{k}{n}\right) E Y_{k}^{2}}{n x_{n}^{2} B_{n}^{2} P\left(X \geq x_{n} B_{n}\right)} \leq \frac{C_{3}}{n} \sum_{k=1}^{n} f^{\alpha-\delta_{0}}\left(\frac{k}{n}\right)$. Since $f$ is continuous $\mathrm{BV}[0,1]$, then there exists $\mathrm{C}_{4}\left(>\mathrm{C}_{3}\right)$ such that

$$
\frac{\sum_{k=1}^{n} f^{2}\left(\frac{k}{n}\right) E Y_{k}^{2}}{n x_{n}^{2} B_{n}^{2} P\left(X \geq x_{n} B_{n}\right)} \leq C_{4} .
$$

Observe that

$$
\sum_{k=1}^{n} \sum_{m=1}^{n} f\left(\frac{k}{n}\right) f\left(\frac{m}{n}\right) E Y_{k} E Y_{m} \leq\left\{\sum_{k=1}^{n} f\left(\frac{k}{n}\right)\left|E Y_{k}\right|\right\}^{2} .
$$

Now for $0<\alpha<1$,

$$
\left|E Y_{k}\right| \leq E\left|Y_{k}\right|=\int_{|x| \leq \frac{X_{n} B_{n}}{f\left(\frac{k}{n}\right)}}|x| d P(X<x) \leq \int_{0}^{\frac{x_{n} B_{n}}{f\left(\frac{k}{n}\right)}} P(X \geq x) d x
$$$$
\text { Let } A=\frac{\sum_{k=1}^{n} \sum_{m=1}^{n} f\left(\frac{k}{n}\right) f\left(\frac{k}{n}\right) E Y_{k} E Y_{m}}{n x_{n}^{2} B_{n}^{2} P\left(X \geq x_{n} B_{n}\right)} \text {, }
$$$$
B=\frac{\left(\sum_{k=1}^{n} f\left(\frac{k}{n}\right)\left|E Y_{k}\right|\right)^{2}}{n x_{n}^{2} B_{n}^{2} P\left(X \geq x_{n} B_{n}\right)} \text { and }
$$

$$
D=\frac{\left(\sum_{k=1}^{n} f\left(\frac{k}{n}\right) \int_{0}^{\frac{x_{n} B_{n}}{f\left(\frac{k}{n}\right)}} P(X \geq x) d x\right)^{2}}{n x_{n}^{2} B_{n}^{2} P\left(X \geq x_{n} B_{n}\right)} .
$$

Notice that $\mathrm{A} \leq \mathrm{B} \leq \mathrm{D}$. Again using Lemma 2.1, we have

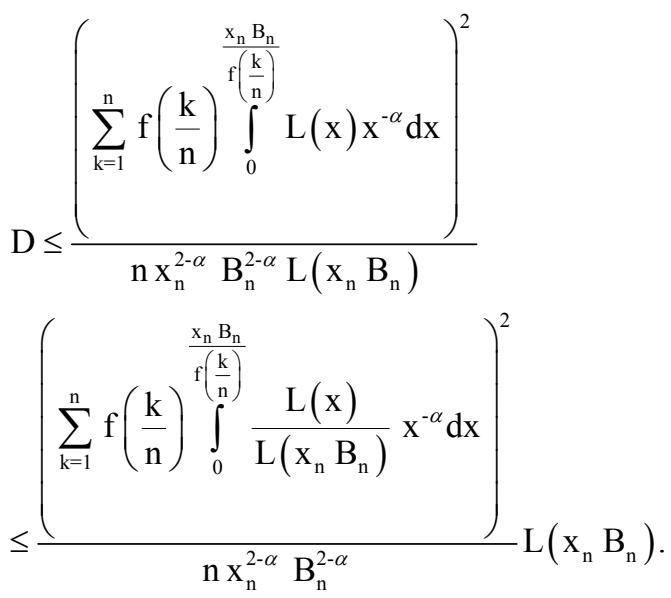


Following similar steps of (4), we can find some constant $\mathrm{C}_{5}$ and $\delta_{0}>0$ such that

$$
\frac{\mathrm{L}(\mathrm{x})}{\mathrm{L}\left(\mathrm{x}_{\mathrm{n}} \mathrm{B}_{\mathrm{n}}\right)} \leq \mathrm{C}_{5}\left(1+\delta_{0}\right)\left(\frac{\mathrm{x}_{\mathrm{n}} \mathrm{B}_{\mathrm{n}}}{\mathrm{x}}\right)^{\delta_{0}} .
$$

Hence

$$
\mathrm{D} \leq \frac{\left(\mathrm{C}_{5}\left(1+\delta_{0}\right) \sum_{\mathrm{k}=1}^{\mathrm{n}} \mathrm{f}\left(\frac{\mathrm{k}}{\mathrm{n}}\right) \int_{0}^{\left.\frac{\mathrm{x}_{\mathrm{n}} \mathrm{B}_{\mathrm{n}}}{\mathrm{f}\left(\frac{\mathrm{k}}{\mathrm{n}}\right)} \mathrm{x}^{-\alpha-\delta_{0}} \mathrm{dx} \mathrm{x}_{\mathrm{n}}^{\delta_{0}} \mathrm{~B}_{\mathrm{n}}^{\delta_{0}}\right)^{2}}\right.}{\mathrm{n} \mathrm{x}_{\mathrm{n}}^{2-\alpha} \mathrm{B}_{\mathrm{n}}^{2-\alpha}} .
$$

Also

$\int_{0}^{\frac{x_{n} B_{n}}{f\left(\frac{k}{n}\right)}} x^{-\alpha-\delta_{0}} d x=\frac{1}{1-\alpha-\delta_{0}} x_{n}^{1-\alpha-\delta_{0}} B_{n}^{1-\alpha-\delta_{0}} f^{\alpha+\delta_{0}-1}\left(\frac{k}{n}\right)$ and there exists $\mathrm{C}_{6}\left(>\mathrm{C}_{5}\right)$ such that

$$
\mathrm{D} \leq \frac{\mathrm{C}_{6}\left(\sum_{\mathrm{k}=1}^{\mathrm{n}} \mathrm{f}^{\alpha+\delta_{0}}\left(\frac{\mathrm{k}}{\mathrm{n}}\right)\right)^{2} \mathrm{~L}\left(\mathrm{x}_{\mathrm{n}} \mathrm{B}_{\mathrm{n}}\right)}{\mathrm{nx}_{\mathrm{n}}^{\alpha} \mathrm{B}_{\mathrm{n}}^{\alpha}} .
$$

Let $\mathrm{M}_{\mathrm{n}}=\mathrm{x}_{\mathrm{n}} \mathrm{B}_{\mathrm{n}}$, where $\mathrm{x}_{\mathrm{n}} \rightarrow \infty$ and $\mathrm{B}_{\mathrm{n}} \rightarrow \infty$ as $\mathrm{n} \rightarrow \infty$. Since $\mathrm{F} \in \mathrm{DP}(\alpha), 0<\alpha<1$, then

We know that

$$
\frac{\mathrm{nL}\left(\mathrm{M}_{\mathrm{n}}\right)}{\mathrm{M}_{\mathrm{n}}^{\alpha}} \rightarrow \mathrm{C}_{7}
$$

Using (8) one can find some constant $\mathrm{C}_{8}$ such that

$$
\mathrm{D} \leq \frac{\mathrm{C}_{8}\left(\sum_{\mathrm{k}=1}^{\mathrm{n}} \mathrm{f}^{\alpha+\delta_{0}}\left(\frac{\mathrm{k}}{\mathrm{n}}\right)\right)^{2}}{\mathrm{n}^{2}} \text {. Since } \mathrm{f} \text { is Continuous BV }[0,1] \text {, }
$$
$\mathrm{n}^{2}$
therefore there exists $\mathrm{C}_{9}$ such that $\sum_{\mathrm{k}=1}^{\mathrm{n}} \mathrm{f}^{\alpha+\delta_{0}}\left(\frac{\mathrm{k}}{\mathrm{n}}\right) \leq \mathrm{nC}_{9}$
and hence

$$
\mathrm{D} \leq \mathrm{C}_{9} \Rightarrow \mathrm{B} \leq \mathrm{C}_{9} \Rightarrow \mathrm{A} \leq \mathrm{C}_{9}
$$

From (7) and (9), we claim that

$\frac{\mathrm{P}\left(\mathrm{T}_{1, \mathrm{n}} \geq \mathrm{x}_{\mathrm{n}} \mathrm{B}_{\mathrm{n}}\right)}{\mathrm{nP}\left(\mathrm{X} \geq \mathrm{x}_{\mathrm{n}} \mathrm{B}_{\mathrm{n}}\right)} \rightarrow 0$ as $\mathrm{n} \rightarrow \infty$, i.e., holds.

Substituting (5) and (6) in (2), we get

$\operatorname{Lim}_{n \rightarrow \infty} \operatorname{Sup} \frac{P\left(T_{n} \geq x_{n} B_{n}\right)}{n P\left(X \geq x_{n} B_{n}\right)}<\infty$. The proof of the theorem is completed.

\section{Chover's Form of LIL}

\section{Theorem 4.1}

Let $\mathrm{F} \in \mathrm{DP}(\alpha), 0<\alpha<1$. Then
$\operatorname{Lim}_{n \rightarrow \infty} \operatorname{Sup}\left(\frac{T_{n}}{B_{n}}\right)^{\frac{1}{\log \log n}}=e^{\frac{1}{\alpha}}$ a.s .

Proof

To prove the assertion, it suffices to show for any $\varepsilon \in$ $(0,1)$, that

$$
\mathrm{P}\left(\mathrm{T}_{\mathrm{n}} \geq \mathrm{B}_{\mathrm{n}}(\log \mathrm{n})^{\frac{1+\varepsilon}{\alpha}} \text { i.o }\right)=0
$$

and

$$
\mathrm{P}\left(\mathrm{T}_{\mathrm{n}} \geq \mathrm{B}_{\mathrm{n}}(\log \mathrm{n})^{\frac{1-\varepsilon}{\alpha}} \text { i.o }\right)=1
$$

To prove (10), let

$A_{n}=\left\{T_{n} \geq B_{n}(\log n)^{\frac{1+\varepsilon}{\alpha}}\right\}$ and $x_{n}=B_{n}(\log n)^{\frac{1+\varepsilon}{\alpha}}$. By the above Theorem 3.1, one can find a $\mathrm{C}_{10}$ such that, $\mathrm{P}\left(\mathrm{A}_{\mathrm{n}}\right) \leq \mathrm{C}_{10} \mathrm{nP}\left(\mathrm{X} \geq \mathrm{x}_{\mathrm{n}}\right)$. Using Lemma 2.1,

$$
\begin{aligned}
& \mathrm{P}\left(\mathrm{A}_{\mathrm{n}}\right) \leq \mathrm{C}_{10} \mathrm{nx}_{\mathrm{n}}^{-\alpha} \mathrm{L}\left(\mathrm{x}_{\mathrm{n}}\right) \\
& \leq \mathrm{C}_{10} \mathrm{n} \frac{\mathrm{L}\left(\mathrm{B}_{\mathrm{n}}\right)}{\mathrm{B}_{\mathrm{n}}^{\alpha}(\log \mathrm{n})^{(1+\varepsilon)}} \frac{\mathrm{L}\left(\mathrm{x}_{\mathrm{n}}\right)}{\mathrm{L}\left(\mathrm{B}_{\mathrm{n}}\right)} .
\end{aligned}
$$

Applying Lemma 2.3 with $\delta=\frac{\varepsilon}{2}$ and using the boundedness of $\theta, \mathrm{P}\left(\mathrm{A}_{\mathrm{n}}\right) \leq \mathrm{C}_{11}(\log \mathrm{n})^{-\left(1+\frac{\varepsilon}{2}\right)}$ for some $C_{11}>0$. Consequently $\sum_{n=1}^{\infty} P\left(A_{n}\right)<\infty$ and (3) follows from the Borel-Cantelli Lemma.

Define, for large $\mathrm{k}$,

$$
\mathrm{m}_{\mathrm{k}}=\min \left\{\mathrm{j}: \mathrm{n}_{\mathrm{j}} \geq \beta^{(\mathrm{k}-1)^{\delta}}\right\},
$$

where $\beta>1$ and $\delta>0$ and from the relation $\mathrm{T}_{\mathrm{n}_{\mathrm{k}}}=\mathrm{T}_{\mathrm{n}_{\mathrm{k}}}-\mathrm{T}_{\mathrm{n}_{\mathrm{k}-1}}+\mathrm{T}_{\mathrm{n}_{\mathrm{k}-1}}, \mathrm{k} \geq 1$, and in order to establish (11), it is enough if we show that $\varepsilon \in(0,1)$, that

$$
\mathrm{P}\left(\mathrm{T}_{\mathrm{n}_{\mathrm{m}_{\mathrm{k}}}}-\mathrm{T}_{\mathrm{n}_{\mathrm{m}_{\mathrm{k}}}} \geq 2 \mathrm{~B}_{\mathrm{n}_{\mathrm{m}_{\mathrm{k}}}}\left(\log \mathrm{n}_{\mathrm{m}_{\mathrm{k}}}\right)^{\frac{1-\varepsilon}{\alpha}} \text { i.o }\right)=1
$$

and

$$
P\left(T_{n_{m_{k}-1}} \geq B_{n_{m_{k}}}\left(\log n_{m_{k}}\right)^{\frac{1-\varepsilon}{\alpha}} \text { i.o }\right)=0
$$

Define $\mathrm{z}_{\mathrm{n}}=\mathrm{B}_{\mathrm{n}}(\log \mathrm{n})^{\frac{(1-\varepsilon)}{\alpha}}$ and

$D_{k}=\left(T_{n_{m_{k}}}-T_{n_{m_{k-1}}}\right) \geq z_{n_{m_{k}}}, k \geq 1$. Note that

$\mathrm{T}_{\mathrm{n}_{\mathrm{m}_{k}}}-\mathrm{T}_{\mathrm{n}_{\mathrm{m}_{\mathrm{k}}-1}} \stackrel{\mathrm{d}}{=} \mathrm{T}_{\mathrm{n}_{\mathrm{m}_{\mathrm{k}}}-\mathrm{n}_{\mathrm{m}_{\mathrm{k}-1}}}, \mathrm{k} \geq 1$. By the above Theorem 3.1, one can find a constant $\mathrm{C}_{12}>0$ and $\mathrm{k}_{1}$ such that for all $\mathrm{k}\left(\geq \mathrm{k}_{1}\right)$,

$$
\begin{aligned}
& P\left(D_{k}\right) \geq C_{12}\left(n_{m_{k}}-n_{m_{k-1}}\right) P\left(X \geq 2 z_{n_{m_{k}}}\right) \\
& =C_{12} n_{m_{k}}\left(1-\frac{n_{m_{k-1}}}{n_{m_{k}}}\right) P\left(X \geq 2 z_{n_{m_{k}}}\right)
\end{aligned}
$$


Since $\mathrm{F} \in \mathrm{DP}(\alpha), 0<\alpha<1$ and under Kruglov's [9] setup i.e., $\operatorname{Lim}_{\mathrm{k} \rightarrow \infty} \frac{\mathrm{n}_{\mathrm{k}+1}}{\mathrm{n}_{\mathrm{k}}}=\mathrm{r}(>1)$ implies that there exists $\lambda$ $=\mathrm{r}^{-1}(>1)$ such that

$$
\frac{\mathrm{n}_{\mathrm{m}_{\mathrm{k}-1}}}{\mathrm{n}_{\mathrm{m}_{\mathrm{k}}}}<\lambda<1 \text { for all } \mathrm{k} \geq \mathrm{k}_{1} \text {. }
$$

$$
\mathrm{P}\left(\mathrm{D}_{\mathrm{k}}\right) \geq \mathrm{C}_{13} \mathrm{n}_{\mathrm{m}_{\mathrm{k}}} \mathrm{P}\left(\mathrm{X} \geq 2 \mathrm{z}_{\mathrm{n}_{\mathrm{m}_{\mathrm{k}}}}\right) \text {, for some } \mathrm{C}_{13}>0 \text {. }
$$

Now following the steps similar to those used to get an upper bound of $\mathrm{P}\left(\mathrm{A}_{\mathrm{n}}\right)$, one can find a $\mathrm{k}_{2}$ such that for all $\mathrm{k}\left(\geq \mathrm{k}_{2}\right), \quad \mathrm{P}\left(\mathrm{D}_{\mathrm{k}}\right) \geq \mathrm{C}_{14}\left(\log \mathrm{n}_{\mathrm{k}}\right)^{-\left(1-\frac{\varepsilon}{2}\right)}$, for some $\mathrm{C}_{14}>0$. Hence $\sum_{\mathrm{k}=\mathrm{k}_{5}}^{\infty} \mathrm{P}\left(\mathrm{D}_{\mathrm{k}}\right)=\infty$. In view of the fact that $\mathrm{D}_{\mathrm{k}}$ 's are mutually independent, by applying the Borel-Cantelli Lemma, (13) is established. Observe that

$$
\begin{aligned}
& \mathrm{P}\left(\mathrm{T}_{\mathrm{n}_{\mathrm{m}_{\mathrm{k}}}} \geq \mathrm{B}_{\mathrm{n}_{\mathrm{m}_{\mathrm{k}}}}\left(\log \mathrm{n}_{\mathrm{m}_{\mathrm{k}}}\right)^{\frac{1-\varepsilon}{\alpha}}\right) \\
& =\mathrm{P}\left(\mathrm{T}_{\mathrm{n}_{\mathrm{m}_{\mathrm{k}-1}}} \geq \mathrm{B}_{\mathrm{n}_{\mathrm{m}_{\mathrm{k}-1}-1}} \frac{\mathrm{B}_{\mathrm{n}_{\mathrm{m}_{\mathrm{k}}}}}{\mathrm{B}_{\mathrm{n}_{\mathrm{m}_{\mathrm{k}-1}}}}\left(\log \mathrm{n}_{\mathrm{m}_{\mathrm{k}}}\right)^{\frac{1-\varepsilon}{\alpha}}\right) .
\end{aligned}
$$

Again by Theorem 3.1, one can find a constant $\mathrm{C}_{15}$ and $\mathrm{k}_{3}$ such that for all $\mathrm{k} \geq \mathrm{k}_{3}$,

$$
\begin{aligned}
& \mathrm{P}\left(\mathrm{T}_{\mathrm{n}_{\mathrm{m}_{\mathrm{k}-1}}} \geq \mathrm{B}_{\mathrm{n}_{\mathrm{m}_{\mathrm{k}}}}\left(\log \mathrm{n}_{\mathrm{m}_{\mathrm{k}}}\right)^{\frac{1-\varepsilon}{\alpha}}\right) \\
& \leq \mathrm{C}_{15} \mathrm{n}_{\mathrm{m}_{\mathrm{k}-1}} \mathrm{P}\left(\mathrm{X}_{1} \geq \mathrm{B}_{\mathrm{n}_{\mathrm{m}_{\mathrm{k}}}}\left(\log \mathrm{n}_{\mathrm{m}_{\mathrm{k}}}\right)^{\frac{1-\varepsilon}{\alpha}}\right) .
\end{aligned}
$$

Again following the steps similar to those used to get an upper bound of $\mathrm{P}\left(\mathrm{A}_{\mathrm{n}}\right)$, one can find a $\mathrm{k}_{4}$ such that for all $\mathrm{k}\left(\geq \mathrm{k}_{4}\right)$,

$$
\mathrm{P}\left(\mathrm{T}_{\mathrm{n}_{\mathrm{m}_{\mathrm{k}}-1}} \geq \mathrm{B}_{\mathrm{n}_{\mathrm{m}_{\mathrm{k}}}}\left(\log \mathrm{n}_{\mathrm{m}_{\mathrm{k}}}\right)^{\frac{1-\varepsilon}{\alpha}}\right) \leq \mathrm{C}_{15} \frac{\mathrm{n}_{\mathrm{m}_{\mathrm{k}-1}}}{\mathrm{n}_{\mathrm{m}_{\mathrm{k}}}} \frac{1}{\left(\log \mathrm{n}_{\mathrm{m}_{\mathrm{k}}}\right)^{\left(1-\frac{3 \varepsilon}{2}\right)}}
$$

By (12) we have $n_{m_{k}} \geq \beta^{(k-1)^{\delta}}$ implies $\mathrm{n}_{\mathrm{m}_{\mathrm{k}+1}} \geq \beta^{\mathrm{k}^{\delta}} \geq \mathrm{n}_{\mathrm{m}_{\mathrm{k}}}$ and (15), we have, $\mathrm{n}_{\mathrm{k}+1} \geq \lambda \mathrm{n}_{\mathrm{k}}$. Therefore, $\mathrm{n}_{\mathrm{m}_{\mathrm{k}+1}} \geq \beta^{\mathrm{k}^{\delta}} \geq \mathrm{n}_{\mathrm{m}_{\mathrm{k}}} \geq \lambda \mathrm{n}_{\mathrm{m}_{\mathrm{k}-1}} \Rightarrow \lambda \mathrm{n}_{\mathrm{m}_{\mathrm{k}-1}} \leq \beta^{\mathrm{k}^{\delta}}$ $\Rightarrow \mathrm{n}_{\mathrm{m}_{\mathrm{k}-1}} \leq \frac{1}{\lambda} \beta^{\mathrm{k}^{\delta}}=\lambda_{\mathrm{l}} \beta^{\mathrm{k}^{\delta}}$, where $\lambda_{\mathrm{l}}=\frac{1}{\lambda}$. Hence $\frac{\mathrm{n}_{\mathrm{m}_{\mathrm{k}-1}}}{\mathrm{n}_{\mathrm{m}_{\mathrm{k}}}} \leq \frac{\lambda_{1} \beta^{\mathrm{k}^{\delta}}}{\beta^{(\mathrm{k}-1)^{\delta}}} \simeq \frac{\lambda_{1}}{\beta^{\mathrm{k}^{\delta_{1}}}}$ and $\sum_{\mathrm{k}=\mathrm{k}_{5}}^{\infty} \frac{\mathrm{n}_{\mathrm{m}_{\mathrm{k}-1}}}{\mathrm{n}_{\mathrm{m}_{\mathrm{k}}}} \frac{1}{\left(\log \mathrm{n}_{\mathrm{m}_{\mathrm{k}}}\right)^{\left(1-\frac{3 \varepsilon}{2}\right)}} \leq \lambda_{1} \sum_{\mathrm{k}=\mathrm{k}_{5}}^{\infty} \frac{1}{\beta^{\mathrm{k}^{\delta_{1}}}\left(\log \mathrm{n}_{\mathrm{m}_{\mathrm{k}}}\right)^{\left(1-\frac{3 \varepsilon}{2}\right)}}<\infty$.
Therefore $\mathrm{P}\left(\mathrm{T}_{\mathrm{n}_{\mathrm{m}_{\mathrm{k}-1}}} \geq \mathrm{B}_{\mathrm{n}_{\mathrm{m}_{\mathrm{k}}}}\left(\log \mathrm{n}_{\mathrm{m}_{\mathrm{k}}}\right)^{\frac{1-\varepsilon}{\alpha}}\right.$ i.o $)=0$, which implies the proof of (11) follows from (13) and (14) and the proof of the theorem is completed.

Another direct application of Theorem 3.1 is for the Cesàro sums of index r. Here we may write $\mathrm{f}\left(\frac{\mathrm{k}}{\mathrm{n}}\right)=\frac{\mathrm{A}_{\mathrm{n}-\mathrm{k}}^{\mathrm{r}}}{\mathrm{A}_{\mathrm{n}}^{\mathrm{r}}}$, where $\mathrm{A}_{\mathrm{n}}^{\mathrm{r}}=\frac{\Gamma(\mathrm{n}+\mathrm{r}+1)}{\Gamma(\mathrm{n}+1) \Gamma(\mathrm{r}+1)}$. Using Sterling approximation, we get $\mathrm{A}_{\mathrm{n}}^{\mathrm{r}}=\frac{\mathrm{n}^{\mathrm{r}}}{\Gamma(\mathrm{r}+1)}$ so that $\mathrm{f}\left(\frac{\mathrm{k}}{\mathrm{n}}\right) \sim\left(1-\frac{\mathrm{k}}{\mathrm{n}}\right)^{\mathrm{r}}$. The following result of Vasudeva [11] can be extended to domain of partial attraction of semi stable law and proof follows on similar lines of Theorem 2 , we omit the details.

\section{Theorem 4.2}

Let $\mathrm{F} \in \mathrm{DP}(\alpha), 0<\alpha<1$. Then

$$
\begin{aligned}
& \operatorname{Lim}_{\mathrm{n} \rightarrow \infty} \operatorname{Sup}\left(\frac{\mathrm{T}_{\mathrm{n}}}{\mathrm{B}_{\mathrm{n}}}\right)^{\frac{1}{\log \log }}=\mathrm{e}^{\frac{1}{\alpha}} \text { a.s, where } \\
& \mathrm{T}_{\mathrm{n}}=\sum_{\mathrm{k}=1}^{\mathrm{n}}\left(1-\frac{\mathrm{k}}{\mathrm{n}}\right)^{\mathrm{r}} \mathrm{X}_{\mathrm{k}} \text { and } \mathrm{r}>0 .
\end{aligned}
$$

\section{Acknowledgements}

The author wishes to express his sincere thanks to $U$ G C, New Delhi, for financial support in the form of Major Research Project (F. No: 34-156/2008 (SR)). Also expresses his regards to the referee for his valuable suggestions.

\section{References}

[1] V. M. Kruglov, "On the Extension of the Class of Stable Distributions," Theory of Probability and Its Applications, Vol. 17, 1972, pp. 685-694. doi:10.1137/1117081

[2] C. C. Heyde, "A Contribution to the Theory of Large Deviations for Sums of Independent Random Variables," Probability Theory and Related Fields, Vol. 7, No. 5, 1967, pp. 303-308. doi:10.1007/BF00535016

[3] C. C. Heyde, "On Large Deviation Problems for Sums of Random Variables Which Are Not Attracted to the Normal Law," The Annals of Mathematical Statistics, Vol. 38, No. 5, 1967, pp. 1575-1578. doi:10.1214/aoms/1177698712

[4] C. C. Heyde, "On Large Deviation Probabilities in the Case of Attraction to a Non-Normal Stable Law," Sankhyā: The Indian Journal of Statistics, Series A, Vol. 30, 1968, No. 3, pp. 253-258.

[5] J. Chover, "A Law of the Iterated Logarithm for Stable Summands," Proceedings of the American Mathematical, 
Vol. 17, No. 2, 1966, pp. 441-443.

[6] L. Peng and Y. C. Qi, "Chover-Type Laws of the Iterated Logarithm for Weighted Sums," Statistics and Probability Letters, Vol. 65, No. 4, 2003, pp. 401-410.

doi:10.1016/j.spl.2003.08.009

[7] G. Divanji, "Law of the Iterated Logarithm for Subsequences of Partial Sums Which are in the Domain of Partial Attraction of Semi Stable Law," Probability and Mathematical Statistics, Vol. 24, pp. 433-442.

[8] G. Divanji and R. Vasudeva. "Tail Behavior of Distributions in the Domain of Partial Attraction and Some Related Iterated Logarithm Laws," Sankhyā: The Indian Journal of Statistics, Series A, Vol. 51, No. 2, pp. 196-204.
[9] D. Drasin and E. Seneta, "A Generalization of Slowly Varying Functions," Proceedings of the American Mathematical Society, Vol. 96, No. 3, pp. 470-471.

[10] R. Vasudeva and G. Divanji, "Law of Iterated Logarithm for Random Subsequences," Statistics \& Probability Letters, Vol. 12, No. 3, 1991, pp. 189-194. doi:10.1016/0167-7152(91)90076-4

[11] R. Vasudeva, “A Log Log Law for Abel's Sum," Acta Mathematica Academiae Scientiarum Hungaricae Tomus Vol. 32, No. 3-4, 1978, pp. 205-215.

[12] W. Feller, "An Introduction to Probability Theory and Its Application,” Wiley \& Sons, New York. 1966. 